Characteristics and outcomes for young patients with advanced bladder cancer

\title{
Clinical characteristics and outcomes for young patients with advanced urothelial carcinoma
}

\author{
Ali Cyrus Chehroudi'; Jean-Michel Lavoie ${ }^{2}$; Peter C. Black ${ }^{1}$; Bernhard J. Eigl ${ }^{2}$ \\ ${ }^{1}$ Department of Urologic Sciences, University of British Columbia, Vancouver, BC, Canada; ${ }^{2}$ Division of Medical \\ Oncology, British Columbia Cancer Agency, Vancouver, BC, Canada
}

Cite as: Can Urol Assoc J 2020 July 27; Epub ahead of print.

http://dx.doi.org/10.5489/cuaj.6405

Published online July 27, 2020

$* * *$

\section{Introduction}

Bladder cancer is the fourth most common diagnosed malignancy in North America and is predominantly a disease of the elderly ${ }^{1}$. The majority of bladder cancer comprises localized, noninvasive tumours; however, a small subset will go on to develop metastatic disease. Metastatic bladder cancer is an aggressive entity with median survival ranging between 12-15 months ${ }^{2}$. First-line systemic therapy comprises platinum-gemcitabine or methotrexate-vincristineAdriamycin-platinum combination systemic therapy, with equivalent outcomes. The median age at diagnosis of bladder is approximately 73 ; however, a small minority $(<10 \%)$ are diagnosed below the age of $60^{1}$. Series to date on outcomes of young patients suggest that most present with non-invasive, low-grade disease and require only trans-urethral resection ${ }^{3}$. Data on outcomes of young patients with advanced disease are limited and specifically, there are no reports on how these patients fare on systemic therapy. Sub-group analysis of various clinical trials in metastatic bladder cancer have not identified age as a risk factor for survival or progression ${ }^{4}$. However, a small retrospective series suggested that younger patients who go on to require cystectomy may have faster time to recurrence compared to a random sampling of elderly patients ${ }^{5}$. Therefore, the objective of this study was to determine the demographics and natural history of patients $\leq 55$ years old with metastatic urothelial carcinoma who receive first-line cisplatin-based chemotherapy.

\section{Methods}

The British Columbia Cancer Agency Database was queried for patients aged $\leq 55$ from 2000 until 2018 who received at least 1 cycle of first-line cisplatin-based systemic therapy for metastatic urothelial carcinoma. A retrospective chart review was then carried out for tumour characteristics, details of treatment, treatment response, and various laboratory parameters at diagnosis. Radiologic progression was defined according to RECIST criteria ${ }^{6}$, and clinical progression was taken as per the treating clinician's assessment. Kaplan-Meier analysis with logrank tests was used to compare progression and survival amongst different potential risk factors. 
Statistical analysis was carried out using SPSS software. A p-value less than 0.05 was taken as significant.

\section{Results}

Out of 250 patients who received first-line cisplatin therapy for metastatic urothelial carcinoma, 66 were under age 55. The median age was 51, ranging from 35-55 years (Table 1). The majority were male (80\%) and most tumours were smoking-associated (65\%). Median ECOG score was 1. All patients received cisplatin-gemcitabine, except for 1 who had cisplatin-methotrexatevincristine. Approximately one-third of patients presented with de novo metastatic disease and $45 \%$ had previous cystectomy; however, only 3 patients $(10 \%$ who received cystectomy for MIBC) received peri-operative chemotherapy. For patients without de novo metastatic disease, median time to metastatic disease was 11.4 months. Twenty-four percent had variant histologies with the most common being micropapillary $(n=4)$ and squamous $(n=3)$. Twenty-seven patients had visceral metastases at diagnosis. Median overall survival and progression-free survival were 10.6 and 7.1 months, respectively (Figure 1). Thirty-seven patients had at least a partial response to first-line cisplatin. However, over $40 \%$ of patients $(n=29)$ were unable to complete initial chemotherapy, mostly due to progression $(n=16)$ followed by adverse events $(n=13)$ (Figure 1). The presence of visceral metastases was significantly associated with poorer OS and PFS (7.7 vs. 13.7 months; 4.5 vs. 8.9 months; $\mathrm{p}<0.05$ ) (Table 2 ). There was a trend towards worse OS in patients with $E C O G \geq 2$ (5.2 vs. 13.3 months; $p=0.06)$. Of the laboratory parameters examined, anemia (8.7 vs. 14.8 months; $p=0.09$ ) and hypoalbuminemia (5.7 vs. 12.9 months; $p=0.262$ ) trended towards poorer OS, but were significantly associated with worse PFS.

Twenty-four patients went on to receive salvage chemotherapy after progression (Table $3)$. This comprised mostly taxane-based regimens $(n=14)$, platinum re-challenge $(n=6)$, and immunotherapy or clinical trial $(n=4)$. Patients who received salvage therapy had improved median overall survival (17.3 vs. 8.5 months, $\mathrm{p}<0.05)$. Median PFS for salvage therapy was 3.4 months for all regimens.

\section{Discussion}

This is one of the first studies to report on the outcomes of young patients with metastatic bladder cancer. Our results suggest that younger patients may have worse outcomes compared to the overall disease population. Data from previous clinical trials as well as large database series report a median overall survival ranging from 14-16 months with first-line platinum-based chemotherapy. In contrast, the median overall survival for our cohort was only 10.6 months, despite being 10 years younger than the Van der Maase trial ${ }^{2}$. On the other end, SEER data looking at elderly patients ( $\geq 66$ years) reveals an overall survival of 12 months ${ }^{7}$. Indeed, one would expect outcomes for younger patients to be at least equivalent to the overall metastatic bladder cancer population given improved performance status, decreased co-morbidities, and perhaps improved tolerance of systemic therapy. Despite this, a majority of patients in our cohort 
were unable to complete first-line platinum therapy and had poorer outcomes than other studies looking at very elderly patients.

Data so far on young patients with bladder cancer is limited to small retrospective series that include mostly patients with non-muscle invasive disease. A review of the National Cancer Database comparing young $(<40)$ to older patients with bladder cancer reported that younger patients were more likely to harbor less invasive disease and accordingly had improved overall survival $^{8}$. However, younger patients who required cystectomy were more likely to have nodal and distant metastases. In line with this, Yossepowitch et al. ${ }^{5}$ report poorer recurrence-free survival in patients under 40 years old who require cystectomy. These findings along with our results suggest that there may be two distinct populations of young patients with bladder cancer: (1) the majority with indolent non-muscle invasive disease; and (2) a smaller sub-set with more aggressive features that carry poorer outcomes compared to older patients of similar stage.

A potential mechanism behind these worse outcomes remains unknown. From a basic oncologic perspective, our group of younger patients seem similar to that expected in the general disease population. The cases captured in our study appear to be mostly smoking-associated and only 2 cases were related to other exposures (previous radiation and indwelling catheter). The prevalence of variant histology was also consistent with that seen in the overall population. The youngest patient we identified was 35 , and most were over 50 years of age. Overall, this is concordant with urothelial carcinoma in younger patients still being a result of repeated carcinogen exposure as opposed to sporadic or genetic.

Seiler et al. ${ }^{9}$ examined mutational burden and transcriptomic profiles of patients older and under 50 years of age with muscle-invasive bladder cancer. They report that those $<50$ years had lower mutational burden and less immunogenic tumours. If these findings are extended to the metastatic setting, decreased mutational burden may negatively impact susceptibility to cisplatin, and lower cytokine expression could translate into diminished anti-tumour immune activity in younger patients. The response rate to platinum in our cohort was similar to large clinical trials ${ }^{2}$; however, it was mostly not durable. The finding from Seiler et al.'s study that would be difficult to extrapolate to our population is that younger patients were more likely to have luminal subtype tumours, which are associated with improved prognosis. However, tumours likely undergo further genomic alteration at metastatic transformation, and whether younger patients may harbor more potent driver mutations remains unknown.

The impact of age has been looked at in more detail in metastatic colorectal ${ }^{10}$ and lung cancers $^{11}$. These patients tend to present with more advanced disease; however, survival seems unchanged or improved. Younger patients also have different mutation profiles, including a greater proportion of driver mutations in lung cancer (EGFR) ${ }^{11,12}$. From a psychosocial perspective, younger patients may be less likely to present earlier at the onset of symptoms. Whether these patterns apply to urothelial carcinoma remains unknown.

Visceral metastases, anemia, hypoalbuminemia, and poor performance status were previously established as adverse prognostic predictors using a cohort of patients in phase II 
clinical trials (Bajorin Risk Factors) ${ }^{4}$. We identified visceral metastases as a risk factor for decreased survival in young patients with metastatic bladder cancer on cisplatin-based therapy. The other Bajorin factors were strongly associated with poorer OS, and were often very close to reaching statistical significance. Low albumin and anemia were significantly associated with poorer PFS. Patients who received salvage systemic therapy had improved survival; however, this likely represents a selection bias of those fit enough to be challenged with second-line treatments.

The largest limitation of our data is that we have not included a direct comparison cohort with patients greater than 55 years. However, the outcomes of this group are well defined through clinical trials and larger databases such as SEER ${ }^{13}$. Furthermore, our survival estimates may be over-estimates for young patients because we excluded those who did not receive cisplatin-based chemotherapy (including carboplatin). While most studies used 40 or 50 as the their age cut-off, these were not specifically looking at metastatic disease and we felt additional time would be required for patients to progress to progress to metastatic disease. Furthermore, based on SEER data ${ }^{1}$ for incidence of bladder cancer, age 55 would still encompass the youngest $10 \%$ of all patients.

\section{Conclusions}

Metastatic urothelial carcinoma in young patients appears to be an aggressive entity with poor survival, at least comparable to elderly patients. Many are unable to complete systemic therapy and decline rapidly after first progression. Known risk factors for survival were also validated in this unique cohort. Larger confirmatory studies and better treatments for this population are needed.

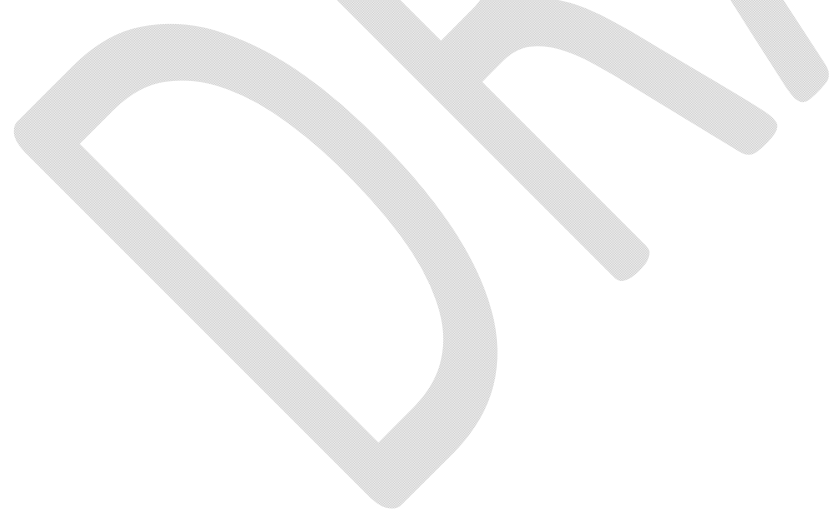




\section{References}

1. https://seer.cancer.gov/statfacts/html/urinb.html

2. Von der Maase H, et al. J Clin Oncol 2000;18(17):3068-77.

3. Stanton ML, et al. Arch Pathol Lab Med 2013; 137:1337-41.

4. Apolo AB, et al. J Natl Cancer Inst 2013; 105(7):499-503.

5. Yossepowitch O, et al. J Urol 2002; 168(1):61-6.

6. Schwartz LH, Litière S, de Vries E, et al. RECIST 1.1 - Update and Clarification: From the RECIST Committee. Eur J Cancer 2016; 62: 132-137.

7. Galsky MD, Pal SK, Lin SW, et al. Real-World Effectiveness of Chemotherapy in Elderly Patients With Metastatic Bladder Cancer in the United States. Bladder Cancer 2018; 4(2): 227-238.

8. De La Calle CM, Washington SL, Meng MV, et al. Young Patients with Bladder Cancer: Outcomes from the National Cancer Database. JACS 2017; 4(S1): S211.

9. Seiler R, Wyatt AW, Black PC. Molecular landscape of carcinoma invading bladder muscle: does patient age matter? BJU Int 2019;124(5):719-721.

10. Vatandoust S, Price TJ, Ullah S, et al. Metastatic Colorectal Cancer in Young Adults: A Study From the South Australian Population-Based Registry. Clin Colorectal Cancer 2016;15(1):32-6.

11. Suidan AM, Roisman L, Belilovski Rozenblum A, et al. Lung Cancer in Young Patients: Higher Rate of Driver Mutations and Brain Involvement, but Better Survival. J Glob Oncol; 2019; 5:1-8.

12. Serebriiskii IG, Connelly C, Frampton G, et al. Comprehensive characterization of RAS mutations in colon and rectal cancers in old and young patients. Nat Commun; 2019;10(1):3722.

13. Galsky MD, Pal SK, Lin SW, et al. Real-World Effectiveness of Chemotherapy in Elderly Patients with Metastatic Bladder Cancer in the United States. Bladder Cancer; 2018; 4(2):227-38. 


\section{Figures and Tables}

Fig. 1. (A). Study results. (B) Median overall survival and progression-free survival.

A

\begin{tabular}{|l|c|}
\hline \multicolumn{1}{|c|}{ First-line Treatment } & Number \\
\hline Cisplatin-Gemcitabine & 65 \\
Methotrexate-Vincristine-Cisplatin & 1 \\
Unable to complete planned therapy & 29 \\
$\quad$ Progression & 16 \\
Adverse Events & 11 \\
\hline
\end{tabular}

B

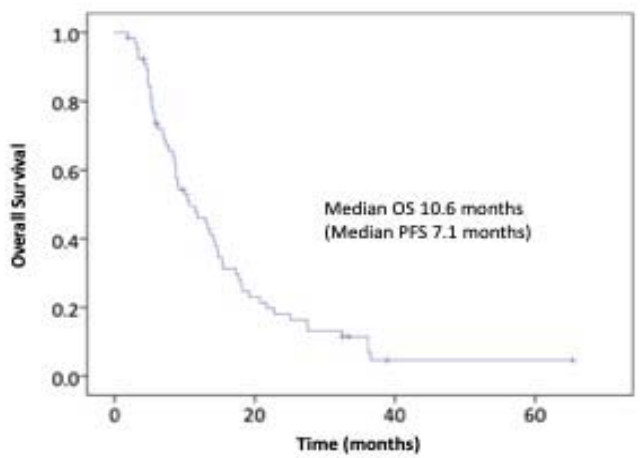

\begin{tabular}{|l|c|}
\hline \multicolumn{2}{|l|}{ Table 1. Patient and tumor characteristics (n=66) } \\
\hline Characteristic & Number (\%) \\
\hline Male gender (\%) & $53(80 \%)$ \\
\hline Median age (years) & 51 years (range 35-55) \\
\hline Smoking-associated & $64(68 \%)$ \\
\hline Variant histology & $16(17 \%)$ \\
\hline Visceral metastases & $27(41 \%)$ \\
\hline ECOG & 21 \\
0 & 29 \\
1 & 11 \\
2 & 5 \\
$\geq 3$ & \\
\hline Best response to systemic therapy & 8 \\
Complete response & 29 \\
Partial response & 6 \\
Stable disease & 22 \\
Progression & $24(36 \%)$ \\
\hline De novo metastatic disease & $30(45 \%)$ \\
\hline Previous cystectomy & $3(10 \%)$ \\
\hline Neoadjuvant or adjuvant chemotherapy for MIBC & $58 \%)$ \\
\hline Deceased at last followup & \\
\hline
\end{tabular}


ECOG: Eastern Cooperative Oncology Group; MIBC: muscle-invasive bladder cancer.

\begin{tabular}{|c|c|c|c|c|}
\hline $\begin{array}{l}\text { Subgroup } \\
\text { Hemoglobir }\end{array}$ & \multicolumn{2}{|c|}{$\begin{array}{c}\text { Median overall survival } \\
\text { (months) }\end{array}$} & \multicolumn{2}{|c|}{$\begin{array}{l}\text { Median progression-free } \\
\text { survival (months) }\end{array}$} \\
\hline$<100$ & 8.7 & & $6.0^{*}$ & \\
\hline$>100$ & 14.8 & $\mathrm{p}=0.094$ & 9.4 & $\mathrm{p}=0.040$ \\
\hline \multicolumn{5}{|c|}{ Visceral metastases } \\
\hline Yes & $7.7 *$ & & $4.5^{*}$ & \\
\hline No & 13.7 & $\mathrm{p}=0.016$ & 8.9 & $\mathrm{p}=0.026$ \\
\hline \multicolumn{5}{|l|}{ Albumin } \\
\hline$<30$ & 5.7 & ( & $3.8^{*}$ & \\
\hline$>30$ & 12.9 & $\mathrm{p}=0.262$ & 8.2 & $\mathrm{p}=0.032$ \\
\hline \multicolumn{5}{|c|}{ De novo metastatic disease } \\
\hline Yes & 10.4 & P & 7.8 & \\
\hline No & 13.0 & $\mathrm{p}=0.063$ & 7.1 & $\mathrm{p}=0.806$ \\
\hline \multicolumn{5}{|l|}{$\mathrm{ECOG}>2$} \\
\hline Yes & 5.2 & & 2.8 & \\
\hline No & 13.3 & $\mathrm{p}=0.055$ & 8.2 & $\mathrm{p}=0.076$ \\
\hline \multicolumn{5}{|c|}{$\begin{array}{l}\text { Use of salvage systemic } \\
\text { therapy }\end{array}$} \\
\hline Yes & 17.3 & & & \\
\hline No & 8.4 & $\mathrm{p}<0.0001$ & & \\
\hline
\end{tabular}

${ }^{*} \mathrm{p}<0.05$ by Log-rank test. 
Characteristics and outcomes for young patients with advanced bladder cancer

\begin{tabular}{|c|c|c|}
\hline Regimen & Number & $\begin{array}{l}\text { Median progression- } \\
\text { free survival (Months) }\end{array}$ \\
\hline Platinum-gemcitabine re-challenge & 6 & 6.8 \\
\hline Taxane monotherapy & 13 & 1.8 \\
\hline Taxane/platinum Combination & 1 & 6.7 \\
\hline Clinical Ttrial & 3 & 4.5 \\
\hline Atezolizumab & 1 & 3.1 \\
\hline Total & 24 & 3.4 \\
\hline
\end{tabular}

